\title{
尺寸排阻-反相液相色谱-质谱联用技术在大鼠肾脏 翻译后修饰蛋白质鉴定中的应用
}

\author{
李健民, 卓 越, 张媇达, 李 娜, 伍建林*
}

(澳门科技大学中医药学院, 中药质量研究国家重点实验室, 澳门 999078)

摘要:LC-MS 联用技术在蛋白质组学研究中具有重要的作用,但是在复杂的生物体系中, 由于样品的高度复杂性和 其中蛋白质含量的巨大差异,执行全面且无倾向的蛋白质组分析是一项挑战。因此,在液相色谱分离中采用基于 不同原理的色谱分离方法来降低蛋白质样本的复杂度,并对微量蛋白质进行富集,对后续采用质谱方法进行信息 的采集和深人分析至关重要。在这里我们开发了一种基于尺寸排阻色谱( SEC) 与反相液相色谱( RPLC)结合的新 方法来进行复杂体系蛋白质的分离和鉴定,特别是对于微量蛋白质的分析。首先使用 SEC 对蛋白质进行分离和富 集,并酶解成多肽,再通过 RPLC-MS 联用的方法对酶解后的多肽进行分离和鉴定。结果显示使用上述方法可以有 效降低蛋白质样本的复杂度, 并有效提高微量蛋白质的鉴定能力, 可从大鼠肾脏鉴定出 23621 个肽段及 1345 个 蛋白质,比常规的二维强阳离子交换-反相液相色谱法 (2D SCX-RPLC) 鉴定到的肽段及蛋白质分别多出 $69 \%$ 及 $27 \%$ 。此外,该方法对肾脏翻译后修饰 (PTM) 蛋白质的鉴定显示出更多的优势, 翻译后修饰的多肽鉴定率显著增 加,特别是磷酸化肽段的鉴定效率可达到靶向富集策略的水平。在此展示的 SEC-RPLC-MS 可以更好地了解蛋白 质翻译后修饰对肾脏的影响,最终将有助于增加我们对正常的生理性肾功能以及病理过程机制的理解。

关键词:蛋白质组学; 尺寸排阻色谱;翻译后修饰

中图分类号: O658 文献标识码:A 文章编号:1000-8713(2021)01-0087-09

\section{Size exclusion-reverse liquid column chromatography-mass spectrometry and its application in the identification of post-translationally modified proteins in rat kidney}

\author{
LI Jianmin, ZHUO Yue, ZHANG Yida, LI Na, WU Jianlin * \\ (Faculty of Chinese Medicine, State Key Laboratory of Quality Research in Chinese Medicine, \\ Macau University of Science and Technology, Macau 999078, China)
}

\begin{abstract}
Proteomics is an emerging field that has been shown to play a crucial role in unveiling the mechanisms underlying physiological and pathological processes, and liquid chromatography-mass spectrometry (LC-MS) is one of the most important methods employed in this field. However, in complex biological systems, such as eukaryotes, it is challenging to perform a comprehensive and unbiased proteome analysis due to the high complexity of biological samples and enormous differences in sample contents. For example, post-translational modifications (PTMs) in proteins are imperative for cell signaling, but post-translationally modified proteins account for about $1 \%$ of the total proteins in a single cell, making their identification extremely difficult. Therefore, chromatographic separation methods based on different principles are generally applied to reduce the complexity of biological samples and enrich trace proteins for their identification through mass spectrometry (MS). In this study, we developed a new proteomics method by combining size exclusion chromatography ( SEC) and reversed-
\end{abstract}




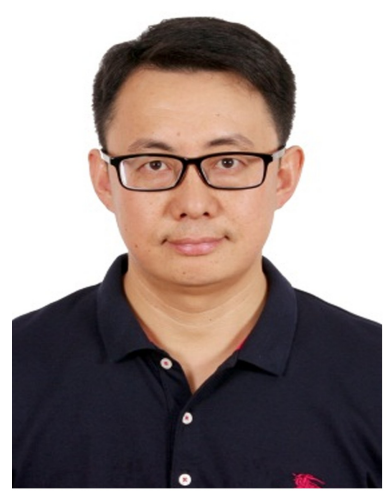

伍建林: 博士, 现任澳门科技大学中药质量研究国家重点实验室副教授, 博士生导 师,广州医科大学南山学者特聘教授(2017-2020)。2004 年获日本新潟大学工学 部工学硕士 (NMR 应用), 2009 年获香港浸会大学化学系哲学博士 ( MS 应用), 后在香港大学李嘉诚医学院从事博士后研究员工作并筹建了基于 LC、MS 和 NMR 的香港大学代谢组学与先端分析实验室,2011 年加入澳门科技大学任助理教授, 筹建了基于 LC、MS 和 NMR 的包括组学技术与创新药物研究中心和澳门核磁共 振与光谱中心。目前主要从事多组学整合分析方法研究消化道肿瘤机制、药物和 食品化学分析及应用等。截至目前, 以第一和通讯作者 (含共同) 在 Cell Res, Gastroenterology, Anal Chem, J Hazard Mater, Environ Int, Food Chem, J Agric Food Chem 和 $J$ Nat Prod 等, 及合作在 $J$ Hepatol, Gastroenterology, Adv $S c i$ 等杂志上发表 LC、MS 和 NMR 应用相关 SCI 文章 80 余篇,授权国际发明专利 5 项。

phase chromatography ( RPLC), to separate and identify trace proteins in complex systems. SEC was used to separate and enrich kidney-specific proteins. After optimization of the method, it was found that $30 \mathrm{mmol} / \mathrm{L}$ of ammonium acetate could efficiently separate rat kidney proteins from the total protein fraction so that they could be eluted based on their relative molecular mass. Sodium dodecyl sulphate-polyacrylamide gel electrophoresis ( SDS-PAGE) analysis and LC-MS results showed that our SEC separation method not only refined the protein composition of the biological sample but also enhanced the relative contents of trace proteins through multiple injections. The collected protein fractions were further concentrated through ultrafiltration centrifugation followed by freeze-drying, which further improved the recovery of trace proteins by approximately $90 \%$ and largely decreased the time required with the use of freezedrying alone. Thereafter, five protein fractions were separately digested using trypsin, and the resultant peptides were further analyzed by reverse phase chromatography-MS analysis. In the RPLC column, the peptides were isolated mainly based on their hydrophobicity. As a result, by combining SEC and RPLC, 23621 peptides and 1345 proteins were identified from the kidney, with an increase in numbers by $69 \%$ and $27 \%$, respectively, when compared to those obtained using the common 2D strong cation exchange ( SCX)-RPLC-MS method. However, no significant difference was observed in the $\mathrm{pI}$ and grand average of hydropathicity (GRAVY) values. Gene ontology ( GO) analysis revealed an increase in the number of proteins in each cell component, especially the membrane. Furthermore, identification of a higher rate of identified peptides than proteins suggested that the protein coverage was also improved, thereby facilitating the detection of PTM proteins. Consequently, five common PTMs in biological processes, including methylation, acetylation, carbamylation, oxidation, and phosphorylation, were examined and compared between the two methods. As expected, the number of post-translationally modified peptides identified using SEC-RPLC-MS were 1.7-1.9 times more than those determined using the SCX-RPLC-MS method. Especially for the identification of phosphorylated peptides, we could achieve the level of the targeted enrichment strategy; however no significant difference was observed in the extents of phosphorylation among serine, threonine, and tyrosine. These results further indicate that upon combining SEC and RPLC, high efficiency could be achieved by decreasing the complexity of the protein sample, and the identification was unbiased. Finally, the phosphorylation of some kidney proteins, such as spectrin, L-lactate dehydrogenase, and ATPases, was found, which is critical for their functions. In summary, the 
SEC-RPLC-MS approach was developed for the identification of rat kidney proteins and is especially applicable for the identification of PTM proteins. Using this method, the identification efficiency for PTM peptides increased significantly. Therefore, this method has potential for better understanding the impact of PTM on kidney proteins and further elucidating the potential mechanisms underlying its physiological and pathological functions.

Key words : proteomics ; size exclusion chromatography ( SEC) ; post-translational modification ( PTM)

蛋白质组学研究近年来引起了越来越多的关 注,并且处于技术发展阶段 ${ }^{[1]}$ 。蛋白质组学的主要 挑战是鉴定高度复杂、宽动态范围样品中的低丰度 蛋白质, 由于样品的复杂性和广泛的动态范围, 执行 无差别的蛋白质组分析是一项非凡的挑战。传统的 一维液相色谱 (1D-LC) 通常无法为复杂样品的分析 提供足够的分离能力 ${ }^{[2,3]}$, 这激发了不同液相色谱 技术 (例如二维液相色谱, 2D-LC) ${ }^{[4]}$ 组合的发展。 在 2D-LC 中, 收集来自第一维液相色谱 (第一液相 色谱) 的流分, 然后将其重新注人第二维液相色谱 (第二液相色谱) ${ }^{[5,6]}$, 两种不同的分离方法相结合 可以获得更高的分离效率 ${ }^{[7]}$ 。如 $2 \mathrm{D}$ 强阳离子交 换-反相液相色谱法 (2D SCX-RPLC), 由于 SCX 色 谱柱上的静电相互作用和 C18 色谱柱上的疏水相 互作用相结合, 具有较高的检测灵敏度和出色的分 离性能, 广泛应用于自下而上的鸟枪法蛋白质组学 当中 ${ }^{[8,9]}$ 。

尺寸排阻色谱法 ( SEC) 是一种广泛用于蛋白 质分离纯化的传统技术, 该技术主要用于制备纯化 和脱盐, 尤其是在分子生物学中, 该技术被大量用于 生产纯净蛋白质 ${ }^{[10-13]}$ 。反相液相色谱( RPLC) 由于 其多功能性、灵活性和耐用性, 是分析多肽及蛋白质 的主要方法之一 ${ }^{[14]}$ 。肽段进人反相色谱柱后, 利用 肽段的疏水性, 根据不同分配系数依次流出, 实现肽 段分离。利用 SEC 对蛋白质进行分离纯化, 不仅可 以降低样品复杂度, 并且可以对复杂蛋白质样品中 的低丰度蛋白质进行富集, 再结合 RPLC 进行肽段 分离实现肽段分析。

蛋白质的翻译后修饰 (PTM) 是具有许多生物 学功能的细胞蛋白质的重要调控机制之一。蛋白质 的翻译后修饰广泛存在于各种细胞器的蛋白质中, 并且在不同种类细胞器中具有不同的生物功能 ${ }^{[15]}$ 。 随着质谱技术的广泛应用, 这些化学修饰在蛋白质 质量上的变化能被精确地检测到, 并且能进行大规 模、全面的 PTM 篮选, 以实现对蛋白质生理研究以
及疾病预防治疗更深人的了解。迄今为止, 已鉴定 出超过 450 种独特的蛋白质修饰,包括磷酸化、乙酰 化、氨基甲酰化、氧化等, 它们可以通过翻译后修饰 改变目标蛋白质的活性、分布、相互作用及寿命 ${ }^{[16]}$ 。 每个细胞当中具有翻译后修饰的蛋白质总量约占单 个细胞总蛋白质的 $1 \%^{[17-20]}$, 且蛋白质浓度的差异 甚至可以达到数百万至数十亿倍, 所以鉴定低浓度 蛋白质及该类蛋白质的翻译后修饰需要更灵敏、准 确的分离及检测方法。肾脏作为重要的排泄器官, 它的基本功能是生成尿液, 借以清除体内代谢产物 及某些废物、毒物, 同时经重吸收功能保留水分及其 他有用物质, 而 PTM 参与肾脏中各种细胞过程和信 号传导途径 ${ }^{[21-27]}$ 。最近的研究表明肾脏缝隙隔膜、 足细胞、神经节和足突的局部粘着可能存在蛋白质 修饰 ${ }^{[22-26,28]}$ 。这些肾脏中的蛋白质修饰, 不仅在肾 小球超滤功能中, 而且在肾稳态调节过程中也起着 关键作用。而磷酸化修饰作为最普遍的 PTM, 几乎 与每个细胞过程密切相关 ${ }^{[29]}$, 它在调节多种代谢酶 的活性中起关键作用 ${ }^{[30]}$, 如协调激活 ATP 合酶为 细胞提供能量维持细胞正常运作等 ${ }^{[31]}$ 。

为了鉴定更多的大鼠肾脏蛋白质以及翻译后修 饰的蛋白质, 我们构建了 SEC-RPLC-MS 系统, 采用 SEC 对肾脏蛋白质样品进行预分离, 降低样品蛋白 质复杂度,并使用 nano-RPLC-MS 对预分离蛋白质 的胰蛋白酶解肽进行分离和鉴定。

\section{1 实验部分}

\section{1 材料与仪器}

醋酸铵、磷酸化盐、乙腈 (均来自美国 SigmaAldrich 公司); 二硫苏糖醇 (DTT), 碘乙酰胺( IAA) 和尿素 (来自美国 GE Healthcare 公司)。胰蛋白酶 (质谱纯) (来自美国 Thermo Fisher Scientific)。 Dionex Ultimate 3000 RSLC UPLC \& Bruker maXis Impact Q-TOF-MS 串联质谱; ST-16R 型高速冷冻 离心机 (美国 Thermo 公司); 水由 Milli-Q 
Ultrapure水系统(美国 Millipore 公司)生产。

\section{2 样品制备}

实验大鼠是购自香港中文大学的 SpragueDawley (SD) 大鼠, 并在澳门科技大学的动物房中 繁殖。选用 8 周龄的雄性 SD 大鼠( $225 \mathrm{~g})$, 麻醉后 生理盐水腹腔主静脉灌注洗净血液, 取出肾脏用磷 酸缓冲盐溶液 $(\mathrm{PBS})$ 洗净。横向切取右肾组织 100 $\mathrm{mg}$, 加人 $1 \mathrm{~mL} P B S$ 匀浆, 离心后取上清液。300 $\mu \mathrm{L}$ 上清液加人 $1200 \mu \mathrm{L}$ 冷丙酮沉淀, $6000 \mathrm{r} / \mathrm{min}$ 下离心 $5 \mathrm{~min}$, 取蛋白质沉淀。蛋白质沉淀加人 300 $\mu \mathrm{L}$ PBS 复溶, $12000 \mathrm{r} / \mathrm{min}$ 下离心 $10 \mathrm{~min}$ 后取 290 $\mu \mathrm{L}$ 上清液待用。

\section{3 肾脏蛋白质 SEC 预分离}

收集肾蛋白质上清液采用 BCA( bicinchoninic acid) 方法测定蛋白质浓度后, 使用 Agilent 1100 system 制备液相系统将 $290 \mu \mathrm{L}(827.5 \mu \mathrm{g})$ 肾蛋白 质溶液进行 SEC 预分离。对于 SEC, 使用 Phenomenex (美国 Torrance 公司) 的 BioSep-SEC3000 色谱柱 ( $300 \mathrm{~mm} \times 7.8 \mathrm{~mm}$ )、Cartridges GFC 3000 保护柱 $(3.0 \mathrm{~mm} \times 4 \mathrm{~mm})$ 。流动相为 30 $\mathrm{mmol} / \mathrm{L}$ 醋酸铵 $(\mathrm{pH}$ 值调至 7$)$, 流速为 $1 \mathrm{~mL} / \mathrm{min}$, 在 $280 \mathrm{~nm}$ 处进行吸光度检测, 依次收集蛋白质流 分。回收的蛋白质流分使用 Millipore 超滤离心管 ( $3 \mathrm{~K}, 15 \mathrm{~mL}$ ) 在 $4000 \mathrm{~g}$ 下离心浓缩 $45 \mathrm{~min}$, 转移 浓缩液至离心管内。冷冻后, 使用冷冻离心干燥机 除去剩余的液体,收集蛋白质粉末。

\section{4 蛋白质酶解}

收集的预分离肾脏蛋白质粉末加人 $100 \mu \mathrm{L} 8$ $\mathrm{mol} / \mathrm{L}$ 尿素进行复溶, $12000 \mathrm{r} / \mathrm{min}$ 下离心 $5 \mathrm{~min}$ 后取上清液,使用 BCA 方法测定蛋白质浓度。取总 蛋白质质量为 $100 \mu \mathrm{g}$ 的上清, 在室温下用 DTT $(200 \mathrm{mmol} / \mathrm{L})$ 还原 $60 \mathrm{~min}$ 。加人 $\mathrm{IAA}(1 \mathrm{~mol} / \mathrm{L})$, 转移至黑暗中孵育 $60 \mathrm{~min}_{\text {。 }}$ 反应混合物在室温下 用 DTT ( $200 \mathrm{mmol} / \mathrm{L}$ ) 淬灭 $60 \mathrm{~min}$ 。加人 25 $\mathrm{mmol} / \mathrm{L}$ 的碳酸铵溶液, 直到尿素浓度低于 1 $\mathrm{mol} / \mathrm{L}$ 。最后, 加人 $2 \mu \mathrm{g}$ 胰蛋白酶, 在 $37{ }^{\circ} \mathrm{C}$ 烘箱消 化过夜。用 SPE C18 柱实现消化液除盐, 洗脱液在 氮气下干燥。

\section{5 nano-RPLC-Q-TOF-MS}

用 $50 \mu \mathrm{L} 2 \%$ 乙腈 (含 $0.1 \%$ 三氟乙酸，v/v) 溶 解胰蛋白酶解肽, $12000 \mathrm{r} / \mathrm{min}$ 下离心 $10 \mathrm{~min}$ 后取 上清液进样。使用 Ultimate 3000 RSLC UPLC ( Dionex) 与 maXis Impact Q-TOF-MS ( Bruker) 串联
系统实现肽段分离和鉴定, 正离子模式使用 Bruker Advance CaptiveSpray 离子源。上清液上样到 Acclaim PepMap 100 nanoViper C18 捕获柱 $(2 \mathrm{~cm}$ $\times 75 \mu \mathrm{m}, 3 \mu \mathrm{m}$, Thermo)。然后在 Acclaim PepMap RSLC nanoViper C18 分析柱 $(15 \mathrm{~cm} \times 75 \mu \mathrm{m}$, $2 \mu \mathrm{m}$, Thermo)上以 $300 \mathrm{~nL} / \mathrm{min}$ 的流速分离肽段。 流动相由 $(\mathrm{A})$ 水中含 $0.1 \%$ 甲酸 $(\mathrm{FA})$ 和 $(\mathrm{B})$ 乙腈中 含 $0.1 \% \mathrm{FA}$ 组成, 梯度如下:0 8.0 min, $5 \% \mathrm{~B} ; 8.0$ $\sim 110.0 \mathrm{~min}, \mathrm{~B}$ 从 $5 \%$ 线性增加到 $40 \% ; 110.0 \sim$ $110.1 \mathrm{~min}, \mathrm{~B}$ 从 $40 \%$ 线性增加到 $80 \% ; 110.1$ $115.0 \mathrm{~min}, 80 \% \mathrm{~B}$; $115.0 \sim 115.1 \mathrm{~min}$, 恢复至 5\% $\mathrm{B} ; 115.1 \sim 120.0 \mathrm{~min}, 5 \% \mathrm{~B}_{\text {。 }}$ 进样量为 $1 \mu \mathrm{L}_{\circ}$ 质 谱用正离子模式下的 Bruker Advance CaptiveSpray 离子源。干燥气的温度为 $160{ }^{\circ} \mathrm{C}$, 流速为 4.0 $\mathrm{L} / \mathrm{min}_{\circ}$ 端板偏移和毛细管电压分别设置为 $500 \mathrm{~V}$ 和 $1400 \mathrm{~V}_{\text {。 }}$ 在 $m / z 330$ 和 1400 之间的范围内选 择了前 10 个前体离子, 并且排除了带单电荷的离 子, 而 $\mathrm{MS} / \mathrm{MS}$ 分析首选带电状态为 $+2 、+3$ 和 +4 的 前体离子。

\section{6 蛋白质鉴定}

使用 Data Analysis 软件处理 MS 数据导出的. mgf 文件,将该. mgf 数据导人 MASCOT 搜索引擎 中, 数据库为 Swiss-Prot 51.6。选择胰蛋白酶作为 酶; 固定修饰为 carbamidomethyl $(\mathrm{C})$, 可变修饰为 Phospho-S、Phospho-T、Phospho-Y、Acetyl-K、 Carbamyl-K、Methyl-DE、Oxidation-M 等 7 个修饰; 前体离子和 $\mathrm{MS} / \mathrm{MS}$ 允许误差范围分别设定为 $30 \times$ $10^{-6}(30 \mathrm{ppm})$ 和 $0.2 \mathrm{Da}$; 肽电荷为 $2^{+} 、 3^{+}$和 $4^{+}$。

\section{2 结果与讨论}

我们了构建 SEC-RPLC 系统,采用 SEC 对肾脏 蛋白质样品进行预分离, 降低样品蛋白质复杂度, 从 而提高低丰度蛋白质的检测灵敏度。

\subsection{SEC 流动相优化及流分浓缩}

\subsubsection{SEC 流动相优化}

为提高尺寸排阻色谱对蛋白质的分离能力, 首 先对流动相进行了优化。20 $\mu \mathrm{L}$ 肾脏蛋白质溶液 (2. $85 \mu \mathrm{g} / \mu \mathrm{L}$ ) 进样, 分别使用 $30 \mathrm{mmol} / \mathrm{L}$ 醋酸铵、 $10 \mathrm{mmol} / \mathrm{L} \mathrm{PBS}$ 或 Milli- $\mathrm{Q}$ 水作为流动相进行分 离, 检测波长为 $280 \mathrm{~nm}_{\text {。 }}$ 在尺寸排阻色谱柱中, 蛋 白质是根据其在流动相中流体动力学体积的差异, 通过扩散进出停滞的孔而分离出来的。具有中等离 子强度的非变性水性缓冲液 (如醋酸铵、磷酸缓冲 
盐溶液等), 通常用于最大程度减少蛋白质与固定 相表面的非特异性相互作用 ${ }^{[32,33]}$ 。PBS 因能有效 提高蛋白质在 SEC 的分离效率而被广泛应用 ${ }^{[34]}$, 但缺点是非挥发性盐的残留在后续的质谱检测时会 导致质谱损伤; 醋酸铵作为挥发性盐被广泛应用到 LC-MS 串联质谱上,可在提高 SEC 蛋白质分离效率 的同时保证蛋白质结构及活性 ${ }^{[35]}$, 且作为挥发性盐 可避免对仪器的损伤。我们的结果表明,使用醋酸 铵的液相色谱图可显示更多的单独色谱峰, 峰形更 完整, 具有更高的分离效率 (见图 1a), 故采用质谱 兼容的醋酸铵作为流动相盐。

接下来, 对流动相的盐浓度进行了优化, 醋酸铵 浓度分别为 $10 、 30$ 和 $50 \mathrm{mmol} / \mathrm{L}$ 。结果显示 30 $\mathrm{mmol} / \mathrm{L}$ 醋酸铵分离效率高于 $10 \mathrm{mmol} / \mathrm{L}$ 醋酸铵, 提示适当提高盐浓度可减少蛋白质表面的电荷数 量,减少蛋白质与填料表面可能的吸附 ${ }^{[36]}$ 。5 $\mathrm{mmol} / \mathrm{L}$ 的分离效果却低于 $30 \mathrm{mmol} / \mathrm{L}$ 醋酸铵的 分离效果 (见图 $1 \mathrm{~b}$ )。虽然缓冲盐能抑制蛋白质与 填料之间的静电效应，但提高盐浓度也会增强蛋白 质与色谱柱填料的疏水相互作用 (通常在高盐浓度 下占主导地位), 影响蛋白质分离效果 ${ }^{[36,37]}$ 。为了 最佳的分离效率,及减少盐浓度便于后续实验处理,
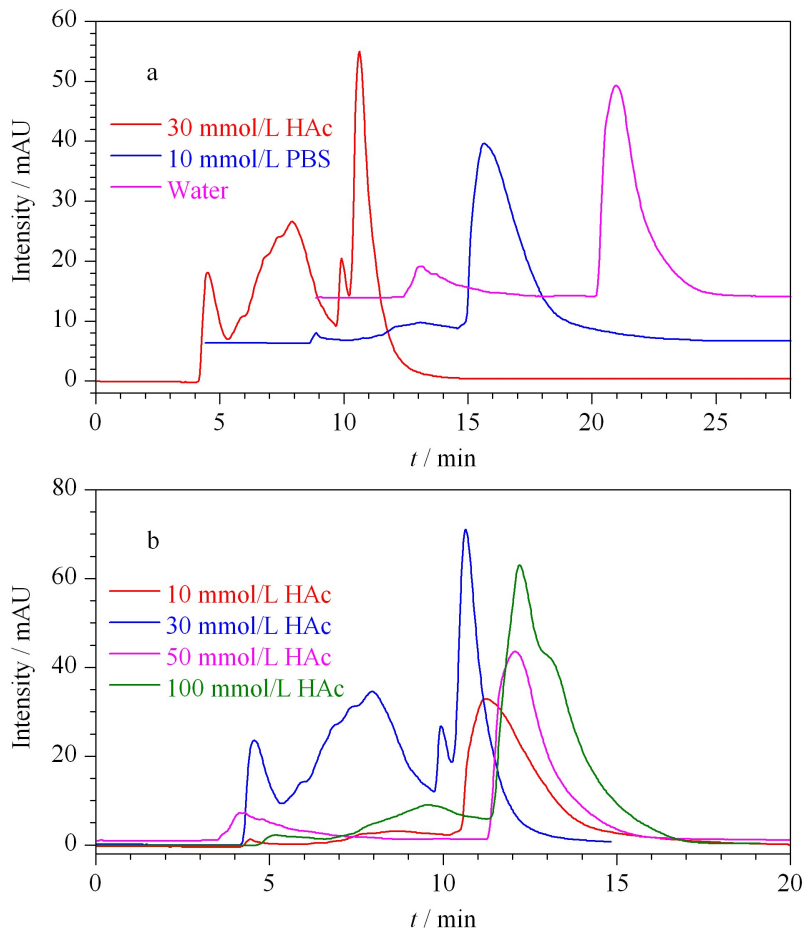

图 1 尺寸排阻色谱流动相的优化

Fig. 1 Optimization of size exclusion chromatography ( SEC) mobile phase

a. buffers; b. concentrations of buffer.

HAc: ammonium acetate; PBS: phosphate buffer solution.
采用 $30 \mathrm{mmol} / \mathrm{L}$ 醋酸铵作为流动相。

\subsection{2 蛋白质流分的浓缩}

在 SEC 分离肾脏蛋白质前及浓缩富集后,分别 测定蛋白质的量并计算回收率。常规的流分浓缩方 法是收集流分后进行冷冻干燥, 回收率仅为 $75.74 \%$, 其可能由于样品较大的体积, 在处理过程 中与容器壁接触面积较大导致样品损失严重; 并且 该方法所需时间过长, 每次冷冻干燥需要 $20 \mathrm{~h}$ 以 上。为了降低蛋白质损失及减少所需时间, 先使用 超滤离心管 (3 K) 对样品进行浓缩, 将浓缩的蛋白 质溶液进行冷冻干燥, 可将浓缩时间降至 $6 \sim 7 \mathrm{~h}$ 。 使用超滤离心管-冷冻干燥方法的蛋白质回收率可 以达到 $89.67 \%$, 高于仅使用冷冻干燥浓缩的方法 (75. 74\%)。后续实验均采用超滤离心管-冷冻干燥 的方法进行流分浓缩。

\subsection{SEC-RPLC 方法}

\subsection{1 样品复杂度分析}

组织蛋白质组成较血清、细胞蛋白质更为复杂, 在当前的研究中,我们测试了使用 SEC 对组织来源 的蛋白质进行预分流来降低样品复杂性的效率。

为了确定尺寸排阻色谱的分离效果, 经过 SEC 分离的蛋白质流分用尿素复溶后, 进行 SDS-PAGE ( sodium dodecyl sulphate-polyacrylamide gel electrophoresis) 分析。通过 SDS-PAGE (见图 2a) 发现 5 个流分中的蛋白质相对分子质量分布呈阶梯 状下降,表明使用 SEC 可通过蛋白质相对分子质量 大小进行分离, 从而有效降低组织蛋白质样品的复 杂度, 且多次进样分离, 可对低丰度蛋白质进行富 集,有效提高低丰度蛋白质的含量。经过 RPLC-MS 分析后,对各个流分中鉴定的蛋白质相对分子质量 分布进行分析, 可发现蛋白质相对分子质量分布范 围与 SDS-PAGE 结果相似, 呈阶梯状下降, 且各流 分中蛋白质相对分子质量的中位数亦呈梯度下降 $(50 、 818 \sim 39 、 963 \mathrm{Da}$ ) (见图 2b)。这说明 SEC 可 降低蛋白质样品复杂度, 多次进样收集流分可有效 富集低丰度蛋白质。

\subsubsection{SEC-RPLC 提高蛋白质及肽段鉴定效率}

如何在高度复杂的宽动态范围样品中鉴定低丰 度蛋白质是当今蛋白质组学的主要挑战, 在这种宽 动态范围下, 高丰度蛋白质与低丰度蛋白质的浓度 差异极大, 高丰度蛋白质的酶解肽段广泛存在, 导致 该类肽段过度采集,最终使得低丰度蛋白质的肽段 信号被掩盖或忽略。 



图 2 各流分蛋白质的相对分子质量分布

Fig. 2 Relative molecular mass distribution of proteins in each fraction

a. SDS-PAGE of the protein fractions collected from size exclusion chromatography; b. median relative molecular mass of proteins in each fraction.

本文所建立的 SEC-RPLC-MS 方法与平行实验 的 SCX-RPLC-MS 方法 ${ }^{[38]}$ 进行比较, 可以发现 SEC-RPLC 方法中的色谱图响应高达 $2 \times 10^{7}$ 而 SCX-RPLC 仅能达到 $2 \times 10^{6}$ 。将各流分蛋白质数据 合并重复项并去除圥余后,SEC-RPLC 鉴定出 1345 个蛋白质和 23621 个肽段; SCX-RPLC 可鉴定出 1053 个蛋白质和 13935 个肽段, 即 SEC-RPLC 较 SCX-RPLC 可显著提升肽段鉴定数量 (1.69 倍) 及 蛋白质鉴定数量 (1.28 倍) (见图 3 )。

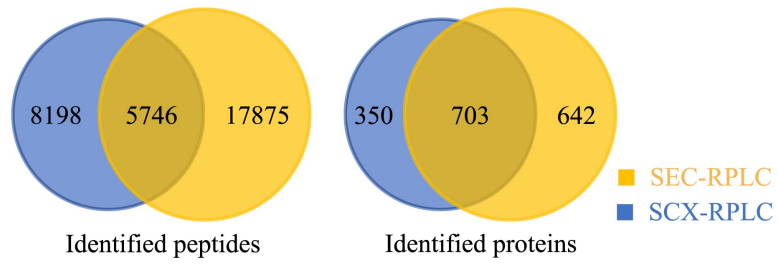

图 3 SEC-RPLC 和 SCX-RPLC 鉴定的蛋白质及肽段数量

Fig. 3 Numbers of proteins and peptides identified by SEC-RPLC and strong cation exchange ( SCX) -RPLC

对蛋白质及肽段的 $\mathrm{pI}$ 值及 GRAVY（grand average of hydropathicity) 值进行比较 (见图 $4 \mathrm{a}$, 4b) , 在 SCX-RPLC 与 SEC-RPLC 方法之间均无显 著性差异, 其符合 SEC 通过蛋白质大小进行分离蛋 白质的物理性质而无倾向性。

对蛋白质鉴定结果进行蛋白质 GO (gene ontology) 分析及分类, 分析结果见图 4c, SECRPLC 各细胞成分蛋白质鉴定数均高于 SCX-RPLC 方法。通过降低蛋白质样品的复杂度, 减少质谱对 高丰度蛋白质肽段的过度采集，提高低丰度蛋白质 的浓度, 最终增加细胞各成分低丰度蛋白质肽段鉴 定的可能性。其中, 膜蛋白质鉴定数有显著提高 （SEC-RPLC：64；SCX-RPLC：15）；虽然该方法在 膜蛋白质的鉴定方面较商业专用试剂盒的鉴定数量 (800 1000) 仍有较大差距, 但商用试剂盒及传统 膜蛋白质提取方法需要 $1 \mathrm{~g}$ 以上的样品, 且多需使 用专属的去污剂对膜蛋白进行提取, 导致非膜蛋白 质变性,无法与膜蛋白质共同检测 ${ }^{[39,40]}$ 。

\subsubsection{SEC-RPLC 对 PTM 修饰蛋白质的鉴定}

蛋白质的翻译后修饰参与调控多种蛋白质的生 物学功能, 但翻译后修饰的蛋白质量仅占单个细胞 蛋白质总量的 $1 \%$, 使其难以被鉴定 ${ }^{[17-19]}$ 。SEC-
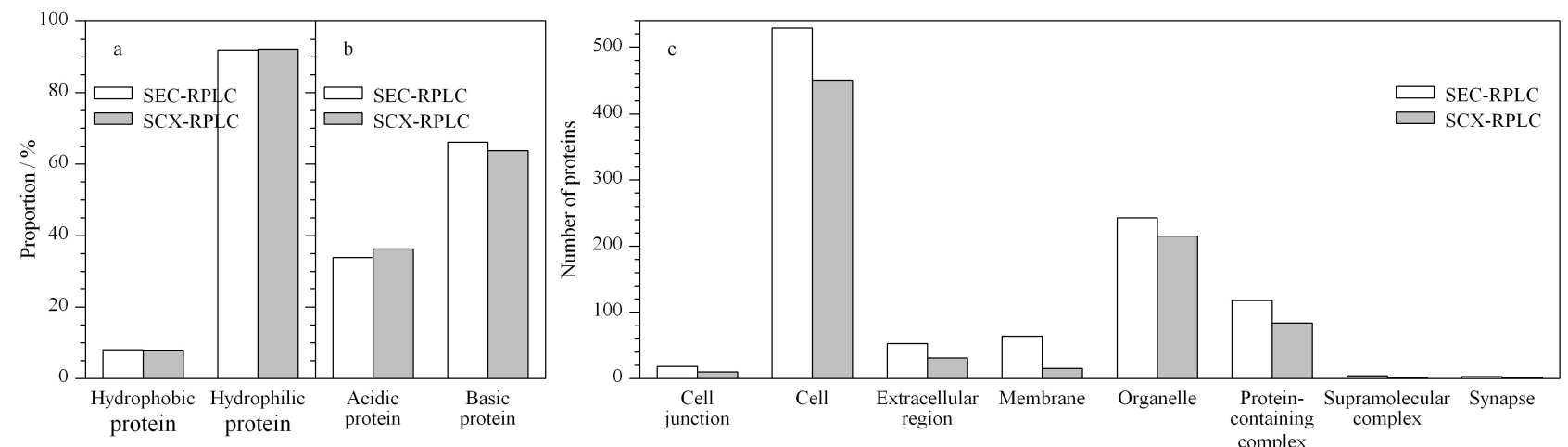

图 4 SEC-RPLC 和 SCX-RPLC 鉴定蛋白质及肽段的物理性质及细胞成分分析

Fig. 4 Physical properties and cell component analysis of proteins and peptides identified by SEC-RPLC and SCX-RPLC a. $\mathrm{pI}$ value; b. GRAVY value; c. cell component. 
RPLC-MS 方法鉴定的肽段数量较 SCX-RPLC-MS 方法提升程度显著,但蛋白质鉴定数增加相对较少, 说明该方法可以提高蛋白质鉴定的覆盖率, 进而可 能使更多潜在的翻译后修饰位点被检测, 从而提高 翻译后修饰被检测的可能性。

为了确定 SEC 预分离技术鉴定潜在 PTM 蛋白 质的能力, 我们比较了使用 SEC-RPLC 及 SCXRPLC 分析的同一组肾脏蛋白质复溶液。如图 5 所 示, 在没有预分离的情况下直接进行分析, SCX-RP 方法鉴定出 9136 个 PTM 肽段,而 SEC-RP 方法鉴 定出 17656 个修饰肽段, 提高了近一倍。

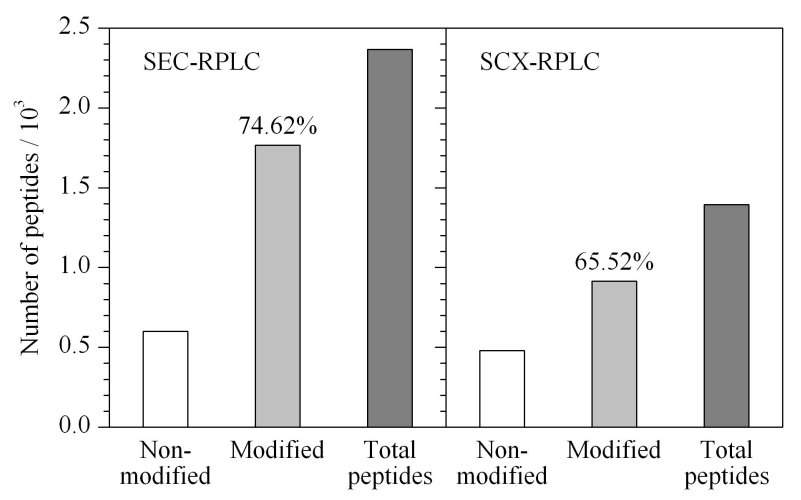

图 5 SEC-RPLC 和 SCX-RPLC 鉴定到的总肽段、 修饰肽段和未修饰肽段的比较

Fig. 5 Comparison of peptides, modified and nonmodified peptides identified by SEC-RPLC and SCX-RPLC

5 种常见翻译后修饰的比较显示 (见图 $6 \mathrm{~b}$ ), 使 用 SEC-RPLC 方法鉴定的最多的修饰是甲基化修 饰肽段为 9599 个, 是 SCX-RPLC 鉴定数目的 1.9 倍; 乙酰化修饰肽段鉴定 5430 个, 是 SCX-RPLC 鉴 定数目的 1.9 倍; 氨基甲酰化修饰肽段鉴定 3553 个, 是 SCX-RPLC 鉴定数目的 1.7 倍。位于蛋氨酸 的氧化修饰肽段鉴定 3636 个, 是 SCX-RPLC 鉴定 数目的 1.9 倍。

与未分离样品 (含磷酸化修饰的蛋白质 838 个)相比,进行预分离的样品鉴定到的含磷酸化修 饰的蛋白质数 (1 171 个) 是 SCX-RPLC 鉴定数目的 1.4 倍(见图 6a)。先前的研究 ${ }^{[41,42]}$ 表明,使用 SCX 或 ERLIC (electrostatic repulsion-hydrophilic interaction chromatography) 进行磷酸化修饰肽分级分 离, 然后进行 IMAC (immobilized metal ion affinity chromatography) 或 $\mathrm{TiO}_{2}$ 纯化, 可以富集 6000 多 种磷酸化修饰肽段。在这里我们在丝氨酸、苏氨酸 或酪氨酸上鉴定了磷酸化修饰的肽段共 6665 个,
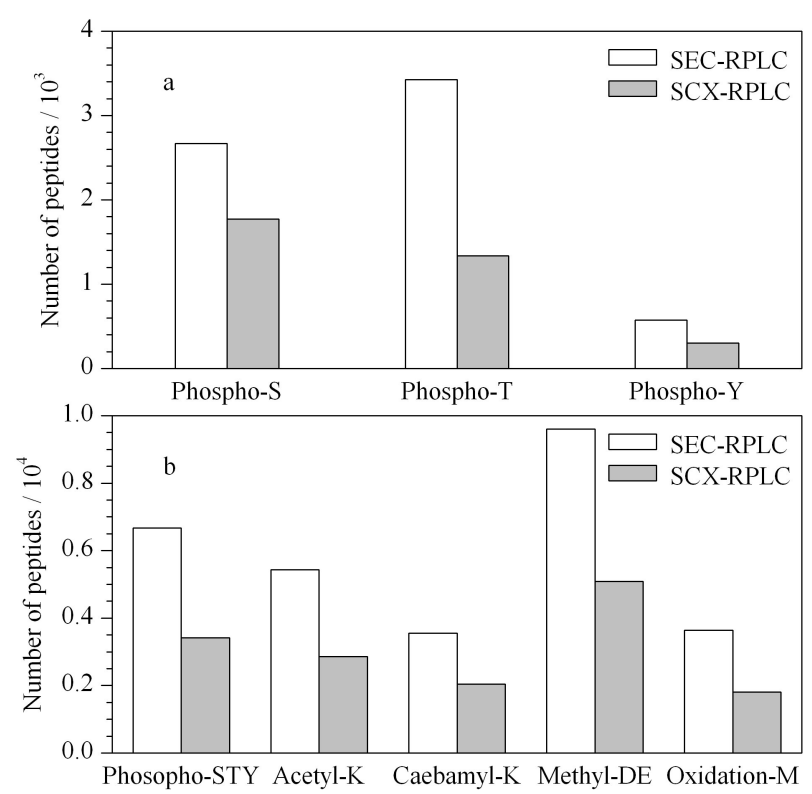

图 6 SEC-RPLC 和 SCX-RPLC 鉴定的大鼠肾脏 翻译后修饰肽段

Fig. 6 Post-translational modified peptides identified from rat kidney by SEC-RPLC and SCX-RPLC

a. phosphorylated modified peptides; b. common posttranslational modified peptides.

而在未分离的蛋白质样品中仅鉴定到 3415 个磷酸 化修饰肽段, 增长近 2 倍。且丝氨酸、苏氨酸、酪氨 酸上修饰的肽段鉴定比例无显著倾向性,这种改进 可能是由于降低了蛋白质样品的复杂性, 从而提高 了在 MS 级别上的鉴定效率。我们的结果表明, SEC 预分离与富集策略相结合, 是鉴定蛋白质修饰 的有力方法, 且对于不同的 PTM 修饰均有作用。

\subsection{4 主要肾脏蛋白质的翻译后修饰研究}

该项研究中,我们发现的多种被修饰的蛋白质 参与了肾脏关键生理功能的调节。我们将以 3 个磷 酸化修饰蛋白质为例进行介绍, 它们是磷酸化的血 影蛋白 (SPTN1_RAT)，L-乳酸脱氢酶 A 链 (LDHA _RAT) 和过渡性内质网 ATP 酶( TERA_RAT)。如 图 7 所示, 血影蛋白的修饰肽段 ${ }^{653}$ MNEVISLWK ${ }^{661}$ 的母离子双电荷峰为 $[\mathrm{M}+2 \mathrm{H}]^{2+} m / z 600.2724$, 比 相应未修饰的肽段多 $79 \mathrm{Da}$, 其中碎片离子 $b_{1} \sim b_{7}$ 、 $y_{1}$ 未被修饰, $y_{6} \sim y_{8}$ 的碎片离子相对分子质量增加 了 $79 \mathrm{Da}$, 结合二级质谱推断被修饰的位点为 S658 (见图 7a)。L-乳酸脱氢酶 $\mathrm{A}$ 链的修饰肽段 ${ }^{318} \mathrm{KSADTLWGIQK}^{328}$ 的母离子双电荷峰为 $[\mathrm{M}+$ $2 \mathrm{H}]^{2+} m / z 670.84800$, 比相应未修饰的肽段多 94 $\mathrm{Da}$, 肽段碎片 $b_{1} \sim b_{3}$ 相对分子质量未增加, $b_{4}$ 相对 分子质量增加了 $14 \mathrm{Da}$, 可推测在 D321 上存在甲基 
化修饰,剩余相对分子质量增加了 $79 \mathrm{Da}$, 可推测存 在磷酸化修饰, 通过二级质谱比对肽段碎片 $b_{1} \sim b_{3}$ 、 $y_{1} 、 y_{3}$ 相对分子质量未增加, 修饰肽段碎片 $b_{7} 、 y_{8} \sim$ $y_{9}$ 的相对分子质量均增加了 $94 \mathrm{Da}$, 由此进一步推 断其被磷酸化修饰的位点为 T322(见图 7b)。过渡 性内质网 ATP 酶的修饰肽段 ${ }^{639}$ LDQLIYIPLPDEK ${ }^{651}$ 的母离子双电荷峰为 $[\mathrm{M}+2 \mathrm{H}]^{2+} m / z 818.89579$, 比 相应未修饰肽段多 $79 \mathrm{Da}$, 其中 $b_{1} \sim b_{4} 、 y_{1} 、 y_{4} \sim y_{6}$ 未 修饰, 修饰肽段 $y_{8} \sim y_{12}$ 的碎片离子相对分子质量增 加了 $79 \mathrm{Da}$, 推断磷酸化修饰位点为 Y644(见图 7c)。

血影蛋白是肾素多蛋白复合物的重要组成部 分, 在肾脏细胞中起到调节膜蛋白质及细胞完整性 的作用 ${ }^{[43]}$; L-乳酸脱氢酶 $\mathrm{A}$ 链参与丙酮酸合成
$(S)$ - 乳酸途径, 从而影响肾脏细胞中 ATP 含量及能 量供给 ${ }^{[44]}$; 过渡性内质网 ATP 酶为囊泡的形成提 供 $\mathrm{ATP}^{[45]}$ 。这些蛋白质经磷酸化后显示出生理活 性 ${ }^{[43,44]}$, 且参与到肾脏的关键生理活性及功能中。 除磷酸化修饰蛋白质外,我们也鉴定出乙酰化、氨基 甲酰化、甲基化、氧化等 4 种蛋白质修饰，已有相关 文献 ${ }^{[46-49]}$ 表明以上修饰与蛋白质的代谢及稳定性 有关, 氧化修饰更可能导致蛋白质活性大变, 并参与 某些疾病的发展。利用 SEC-RPLC-MS 对蛋白质翻 译后修饰进行研究, 可鉴定到参与肾脏关键生理活 性及功能的蛋白质及修饰肽段, 有利于更好地了解 蛋白质修饰, 并有助于我们了解肾脏蛋白质的正常 生理功能及病理机理。
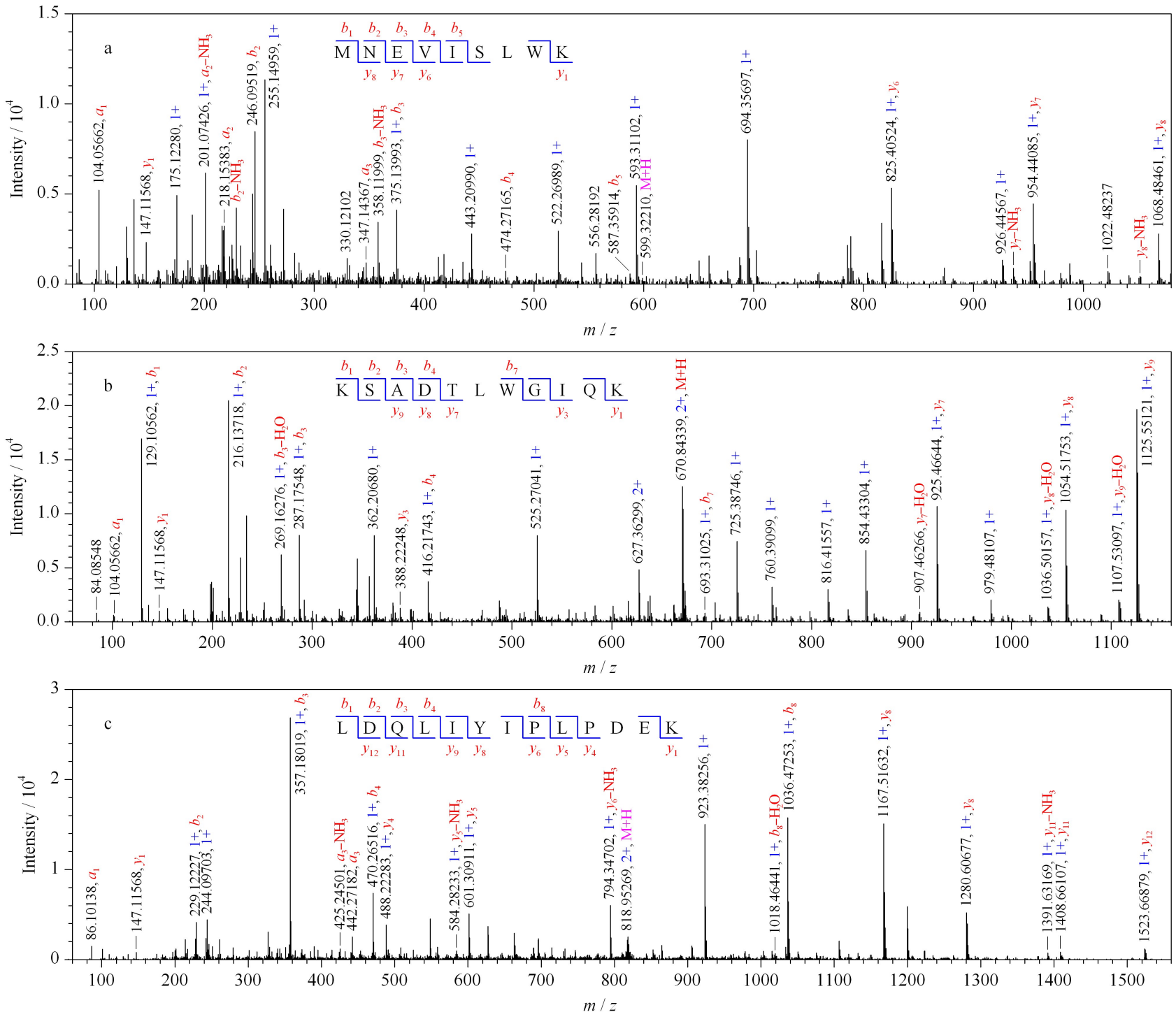

图 7 磷酸化修饰肽段二级质谱图

Fig. 7 MS/MS spectra for phosphorylated peptides

a. SPTN1_RAT Phospho-serine (S) ( $m / z$ 600.27240); b. LDHA_RAT Phospho-threonine ( T) \& Methyl-Aspartic acid (D) ( $m / z$ 670.84800); c. TERA_RAT Phospho-tyrosine $(\mathrm{Y})(\mathrm{m} / z$ 818.89579). 


\section{3 结论}

我们的研究提出了 SEC-RPLC-MS 二维分离系 统, 以鉴定大鼠肾脏蛋白质中的微量蛋白质及翻译 后修饰的蛋白质。此处报告的数据表明, SECRPLC 二维分离系统可显著增强肾脏蛋白质及肽段 的鉴定, 提高了蛋白质覆盖率。我们还展示了通过 SEC-RPLC 预分离技术提高 PTM 鉴定效率的可行 性,特别是对磷酸化肽段的检测。此方法可应用于 大鼠肾脏蛋白质组分析, 更好地了解蛋白质修饰, 并 最终有助于我们解释这些修饰在肾脏的正常生理功 能维持和疾病发生中的潜在作用。

\section{参考文献:}

[1] Harry J L. Electrophoresis, 2015, 21(6) : 1071

[2] Pires R A, Abul-Haija Y M, Costa D S, et al. Anal Chim Acta, 2015, 137(2): 576

[3] Kemmei T, Kodama S, Yamamoto A, et al. Anal Chim Acta, 2017, 958: 71

[4] Li J F, Yan X, Wu Y L, et al. Anal Chim Acta, 2017, 962 : 114

[5] Pursch M, Buckenmaier S. Anal Chim Acta, 2015, 87( 10): 5310

[6] Franois I, Sandra K, Sandra P. Anal Chim Acta, 2009, 641 $(1 / 2): 14$

[7] Bedani F, Kok W T, Janssen H G. Anal Chim Acta, 2009, 654(1): 77

[8] Zhu M Z, Li N, Wang Y T, et al. J Proteome Res, 2017, 16 (9) : 3470

[9] Xie F, Smith R D, Shen Y. J Chromatogr A, 2012, 1261 (20): 78

[10] Burgess R R. Protein Expr Purif, 2018, 150: 81

[11] Tiselius A, Porath J, Albertsson P A. Science, 1963, 141 (3575): 13

[12] Alaa A, Matthew G. Crystals, 2017, 7(11): 331

[13] Zhang P, Mazoyer P, Gilbert R G. J Chromatogr A, 2016, 1443: 267

[14] Wang N, Xie C, Young J B, et al. Anal Chem, 2009, 81 (3) : 1049

[15] Zamaraev A V, Kopeina G S, Prokhorova E A. Trends Cell Biol, 2017, 27: 322

[16] Bah A, Forman-Kay J D. J Biol Chem, 2016, 291 ( 13 ) : 6696

[17] Vu L D, Gevaert K, De SmetI. Trends Plant SCI, 2018, 23 (12) : 1068

[18] Su M G, Weng T Y, Hsu B K, et al. BMC Syst Biol, 2017, $11(\mathrm{~S} 7): 132$

[19] Griffiths J R, Unwin R D. Analysis of Protein Post-Translational Modifications by Mass Spectrometry. New York: John Wiley \& Sons Inc., 2016

[20] Compton P D, Kelleher N L, Jeremy G. J Proteome Res, $2018,17(8): 2727$
[21] Zha D, Chen C, Liang W, et al. BMB Rep, 2013, 46(4): 230

[22] Zhang Y, Yoshida Y, Nameta M, et al. Nephrol Dial Transplant, 2010, 25(6): 1785

[23 ] Nicolaou N, Margadant C, Kevelam SH, , et al. J Clin Invest, 2012, 122( 12) : 4375

[24] Chuang P Y, Dai Y, Liu R, et al. PLoS One, 2011, 6( 8) : e23566

[25] Daehn I S. Front Med, 2018, 5: 113

[26] Togawa A, Yamamoto T, Suzuki H, et al. Am J Pathol, $2003,163(4): 1645$

[27] Isobe S, Ohashi N, Ishigaki S, et al. Hypertens Res, 2016, $39(5): 312$

[28] Yan K, Khoshnoodi J, Ruotsalainen V, et al. Nephrol, $2002,13(5): 1385$

[29] Neiswinger J, Uzoma I, Cox E, et al. Cold Spring Harb Protoc, 2016, 2016(10): 892

[30 ] Grindheim A K, Saraste J, Vedeler A. Biochim Biophys Acta Gen Subj, 2017, 1861(11): 2515

[31] Alain D, Thomas M, Alexander H, et al. Front Physiol, 2018, $9: 329$

[32] Hong P, Koza S, Bouvier ESP. Liq Chromatogr Relat Technol, 2012, 35(20): 2923

[33 ] Alexandre G, Szabolcs F, Alain B, et al. J Chromatogr B, 2018, 1092: 368

[34] Yumioka R, Sato H, Tomizawa H, et al. J Pharm Sci, 2010, 99(2): 618

[35] De Vos J, Kaal E R, Swart R, et al. J Sep Sci, 2016, 39: 689

[36] Tsumoto K, Ejima D, Senczuk A M, et al. J Pharm Sci, $2007,96(7): 1677$

[37] Arakawa T, Ejima D, Li T, et al. J Pharm Sci, 2010, 99 (4) : 1674

[38 ] Yue Z, Wu J L, Yan X, et al. Anal Chem, 2017, 89(24): 13167

[39] Carter P, Smith L. Cancer, 2004, 11: 659

[40] Morrissey K M, Stocker S L, Wittwer M B, et al. Annu Rev Pharmacol Toxicol, 2013, 53: 503

[41] Zarei M, Sprenger A, Metzger F, et al. Proteome Res, $2011,10(8): 3474$

[42] Olsen J V, Blagoev B, Gnad F, et al. Cell, 2006, 127(3): 635

[43] Lehtonen S, Ryan J J, Kudlicka K, et al. Proc Natl Acad Sci U S A, 2005, 102(28) : 9814

[44] Kim J H, Bae K H, Byun J K, et al. Biochem Biophys Res Commun, 2017, 492(1): 41

[45] Roy L, Bergeron J J, Lavoie C, et al. Mol Biol Cell, 2000, 11( 8) : 2529

[46] Ahn B, Coblentz P D, Beharry A W, et al. Front Physiol, 2017, 7: 686

[47] Berlett B S, Stadtman E R. J Biol Chem, 1997, 272( 33 ): 20313

[48] Feng W, Zhang K, Liu Y, et al. Am J Physiol Heart Circ Physiol, 2018, 314(3): H475

[49] Yavuzer S, Yavuzer H, Cengiz M, et al. Aging Clin Exp Res, 2016, 28(4) : 625 